\title{
Reduced Ordering Technique of Impulsive Noise Removal in Color Images
}

\author{
Bogdan Smolka and Krystyna Malik \\ Silesian University of Technology, Department of Automatic Control, \\ Akademicka 16 Str, 44-100 Gliwice, Poland \\ smolka@ieee.org, krystyna.malik@polsl.pl
}

\begin{abstract}
In the paper a fast technique of impulsive noise removal in color images is described. The proposed method is assigning to pixels of the filtering window the sum of the distances to their $k$ nearest neighbors. The difference between the trimmed sum assigned to the central pixel and to the pixel minimizing the cumulated distances is treated as a measure of pixel's distortion caused by the impulsive noise process. If the difference exceeds a global threshold value, then the central pixel of the processing window is replaced by the mean of the pixels from the window, which were found to be not corrupted, otherwise the central pixel is retained. The new filtering design is able to effectively suppress impulsive noise, while preserving fine image details. The performance comparison shows that the proposed filtering design yields significantly better denoising results than the most efficient filters developed for the impulsive noise suppression in color images.
\end{abstract}

Keywords: Color image enhancement, impulsive noise reduction.

\section{Introduction}

Quite often the quality of color images is deteriorated by various types of noise disturbances, whose reduction is indispensable to enable the success of subsequent image processing steps. Noise, arising from a variety of sources, is an undesired feature of the imaging sensors and therefore the distorted signal has to be processed by a filtering algorithm that reduces the noise component, while preserving original image structures [1, 2].

Quite often color images are corrupted by the so called impulsive noise, caused mainly by malfunctioning sensors in the image formation pipeline, faulty memory systems, aging of the storage material or transmission errors due to natural or man-made sources [3]. The suppression of the impulsive noise in color images is the subject of the proposed restoration algorithm.

The color image will be treated as a two-dimensional matrix consisting of $N$ pixels $\boldsymbol{x}_{i}=\left(x_{i 1}, x_{i 2}, x_{i 3}\right)$, where the index $i=1, \ldots, N$ indicates the pixel position on the image domain. The vector components $x_{i k}$, for $k=1,2,3$ represent the RGB color channels values quantified into the integer domain.

Generally, filtering operators work on the assumption that the local image features can be extracted from a small image region centered at pixel $\boldsymbol{x}_{i}$ which

S. Tominaga, R. Schettini, and A. Trémeau (Eds.): CCIW 2013, LNCS 7786, pp. 296-310, 2013.

(C) Springer-Verlag Berlin Heidelberg 2013 
is called a sliding filtering window, $W$. Thus, the output of the filtering operation will depend only on the $n$ samples contained within the moving window centered at the pixel $\boldsymbol{x}_{i}$, which will be also denoted for simplicity as $\boldsymbol{x}_{1}$, $\left(W=\left\{\boldsymbol{x}_{1}, \boldsymbol{x}_{2}, \ldots, \boldsymbol{x}_{n}\right\}\right)$.

The most popular family of nonlinear filters used for impulsive noise removal in color images is based on the order statistics [4]. These filters perform the vector ordering of the set of pixels from the filtering window in order to determine the filtering result.

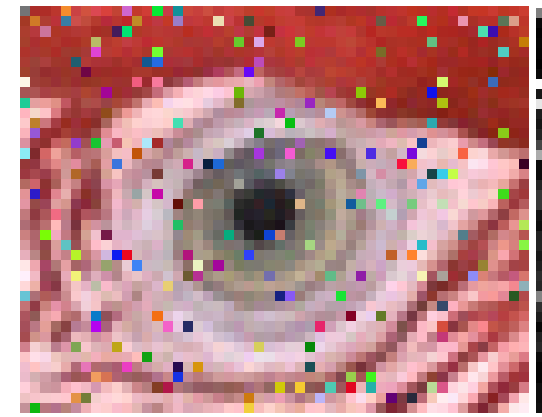

(a) test image

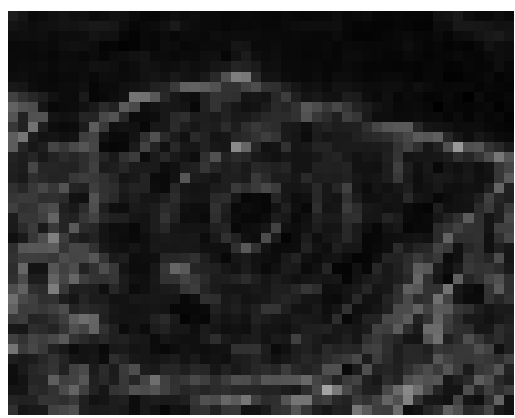

(c) map of $D_{(1)}$

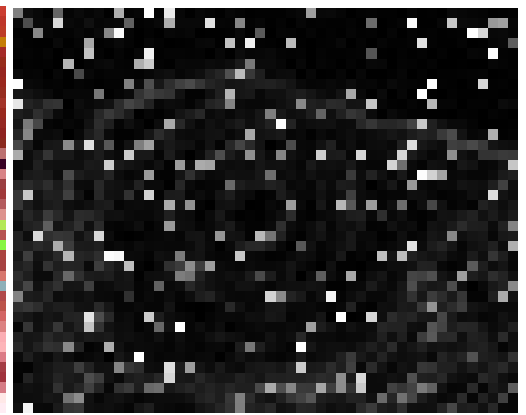

(b) map of $D_{1}$

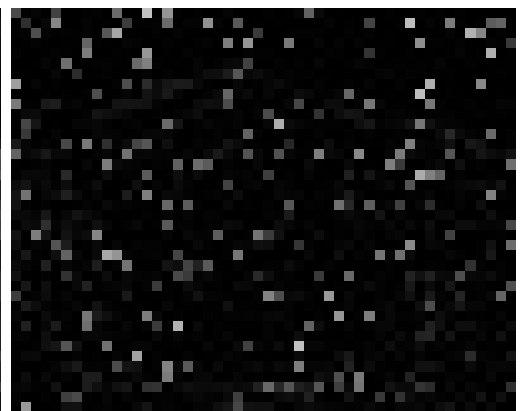

(d) detected noise: $\delta=D_{1}-D_{(1)}$

Fig. 1. Illustration of the noise detection scheme: part of test image LENA (a), map of $D_{1}(\mathrm{~b})$, map of corresponding $D_{(1)}$ values (c) and the image depicting the strength of the impulses: $D_{1}-D_{(1)}$, (d)

The widely used reduced vector ordering is based on assigning a dissimilarity measure to each color pixel from the filtering window [1, 5, 6]. The aggregated dissimilarity measure assigned to pixel $\boldsymbol{x}_{i}$ is defined as

$$
R_{i}=\sum_{j=1}^{n} \rho\left(\boldsymbol{x}_{i}, \boldsymbol{x}_{j}\right), \quad \boldsymbol{x}_{i}, \boldsymbol{x}_{j} \in W,
$$

where $\rho(\cdot)$ denotes the distance between pixels in a given color space. The values of $R_{i},(i=1, \ldots, n)$ are then sorted and the vectors $\boldsymbol{x}_{1}, \boldsymbol{x}_{2}, \ldots, \boldsymbol{x}_{n}$ are correspondingly ordered 


$$
R_{(1)} \leq \ldots \leq R_{(n)} \Rightarrow \boldsymbol{x}_{(1)} \prec \ldots \prec \boldsymbol{x}_{(n)},
$$

where $\prec$ denotes the order relation between vectors and $R_{(r)}$ denotes the $r$-th smallest value of $R$.

Many denoising methods define the vector $\boldsymbol{x}_{(1)}$ in (21) as their output, since vectors that diverge significantly from the samples of $W$ appear in the higher indexed locations in their ordered sequence. Using the Euclidean distance as a dissimilarity measure, the Vector Median Filter (VMF) is obtained [4]. The VMF output $\boldsymbol{x}_{(1)}$ is one of the pixels from the filtering window for which the sum of distances to all other vectors from $W$ is minimized.

The drawback of the VMF and other filters based on vector ordering lies in introducing too much smoothing, which results in an extensive blurring of the output image. This effect is caused by uniform processing of every image pixel, regardless whether it is noisy or not, which leads to the unnecessary replacement of pixels which were not disturbed by the noise process. This effect results in the distortion of image details and generation of color artifacts.

In order to alleviate the problem of excessive image smoothing various switching filters, that replace only the corrupted pixels, have been proposed $7-11]$. The efficiency of a switching filter depends both on the quality of the impulse detection scheme and on the applied restoration framework, which replaces the detected impulses with estimates derived from the samples belonging to a local processing window.

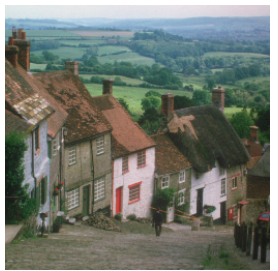

(a) GOLDHILL

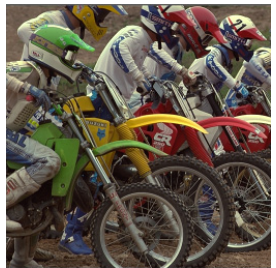

(e) MOTOCROSS

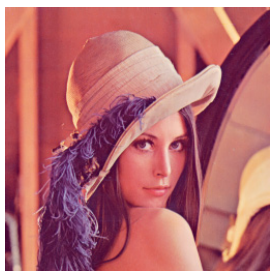

(b) LENA

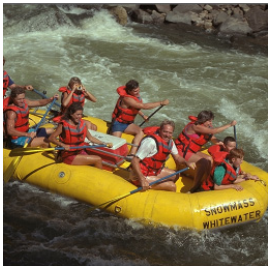

(f) RAFTING

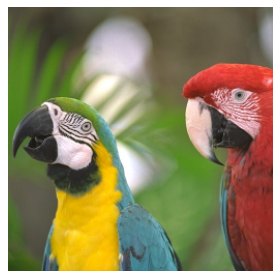

(c) PARROTS

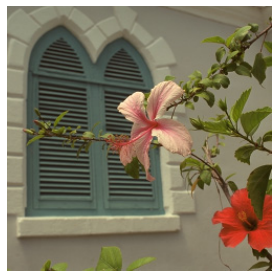

(g) FLOWER

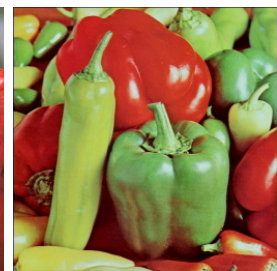

(d) PEPPERS

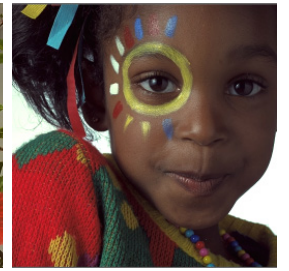

(h) GIRL

Fig. 2. Color test images used for the evaluation of the restoration results

In [8, 9] the cumulated similarity measures were assigned to the color samples from the filtering window. The filter was made adaptive by adjusting its param- 


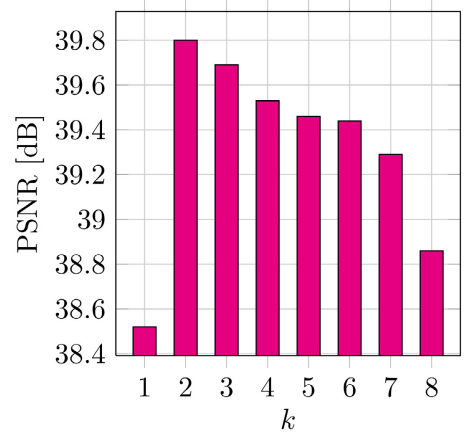

(a) LENA, $p=0.1$

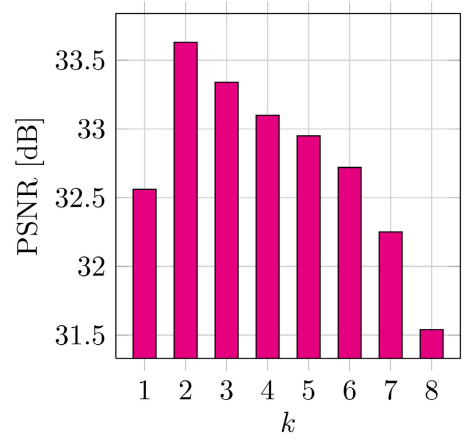

(c) LENA, $p=0.3$

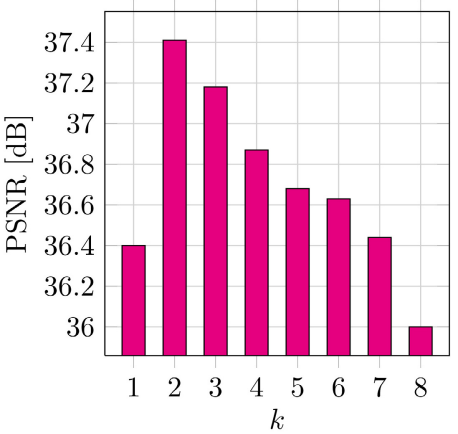

(b) GOLDHILL, $p=0.1$

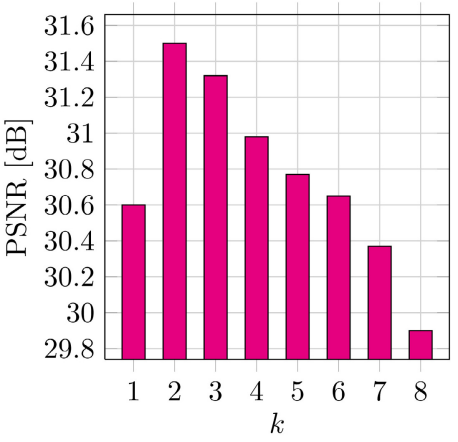

(d) GOLDHILL, $p=0.3$

Fig. 3. Dependence of the optimal PSNR values on the $k$ parameter for the LENA and GOLDHILL color test image distorted by impulsive noise process of intensity $p=0.1$ and $p=0.3$

eter using a simple noise intensity level estimator in which a pixel is considered as not disturbed by the noise process, if there exist at least two pixels whose distance to the central pixel does not exceed a predefined threshold. The concept has been further extended in [10, 12 15].

The sum of the $k$ smallest distances between the central pixel of the filtering window and the remaining pixels was used as a measure of pixel corruption in [16]. This concept is an extension of the statistics introduced in [17].

In [7], the distance between the central pixel of the window and the $\alpha$-trimmed mean was chosen as a dissimilarity measure. If the value of the distance exceeds a predefined threshold, then the central pixel is declared as corrupted and is replaced by VMF, otherwise it is kept unchanged. A filtering scheme, utilizing the angular distance between vectors was presented in [18].

In [19, 20] the concept of the Vector Sigma Filter has been proposed. The switching filtering scheme is utilizing the cumulated Euclidean distances between 
a pixel and all remaining samples belonging to the filtering window as a measure of the dispersion of vectors, which is further used for the detection of outliers introduced by the impulsive noise.

An efficient family of filters utilizing the concept of a peer group was introduced in 21, 22 and extensively used in various filtering designs [19, 10, 23 27]. The peer group associated with the central pixel of a filtering window denotes the set of $k$ close pixels whose distance to the central pixel is not exceeding a predefined threshold. In this way, the pixels are classified as similar to the central pixel or declared as outliers, which should be replaced by a suitable robust filter.

In this paper a novel switching filter based on the peer group concept is proposed. The main advantage of the novel approach is its ability to suppress the noise component, while preserving fine image details. The structure of the filter is based on the reduced ordering statistics and is characterized by a low computational complexity, which enables the adoption of the novel technique in real-time applications.

The paper is structured as follows. In the next Section the proposed filtering technique is described. In the next Section we analyze the influence of the filter's parameter on the effectiveness of the proposed enhancement design. In Section 4 the comparison with the state-of-the-art denoising filters is performed. At the end, some conclusions are drawn.

\section{Proposed Switching Filter Design}

The reduced ordering schemes are based on the sum of the dissimilarity measures between a given pixel and the samples from the filtering window $W$. In this way, the output of the vector median filter is the pixel whose average distance to other pixels is minimized.

The distances $\rho_{i j}=\rho\left(\boldsymbol{x}_{i}, \boldsymbol{x}_{j}\right)$ between the pixel $\boldsymbol{x}_{i}$ and all other pixels $\boldsymbol{x}_{j}$ belonging to $W(j=1, \ldots, n)$ can be be arranged into a sequence

$$
\rho_{i 1}, \rho_{i 2}, \ldots, \rho_{i n} \rightarrow \rho_{i(1)}, \rho_{i(2)}, \ldots, \rho_{i(n)},
$$

where $\rho_{i(r)}$ is the $r$-th smallest distance and $r$ is the distance rank.

The ranks of the ordered distances can be used for the evaluation of the aggregated distances in (1). Instead of the aggregated distances in (1) a weighted sum of distances, utilizing the distance ranks, can be composed

$$
D_{i}=\sum_{r=1}^{n} f(r) \cdot \rho_{i(r)},
$$

where $f(r)$ is a weighting function of the distance rank $r$.

Then, the rank weighted sums of distances can be sorted and a new sequence of vectors is obtained

$$
D_{(1)}, D_{(2)}, \ldots, D_{(n)} \rightarrow \tilde{\boldsymbol{x}}_{(1)}, \tilde{\boldsymbol{x}}_{(2)}, \ldots, \tilde{\boldsymbol{x}}_{(n)},
$$


where the vector $\tilde{\boldsymbol{x}}_{(1)}$ is the output of the Rank Weighted Vector Median Filter (RWVMF) 28-31]. Applying a step-like function

$$
f(r)=\left\{\begin{array}{lc}
1, & \text { for } r \leq k, k \leq n \\
0, & \text { otherwise }
\end{array}\right.
$$

the Sharpening Vector Median Filter (SVMF) presented in [28] is obtained.

The rejection of large distance decreases the influence of the outliers injected by the noise process, which enables to efficiently remove the impulsive noise while enhancing the image edges. Performed experiments revealed that satisfactory denoising results are achieved using $k=2$ or $k=3$.

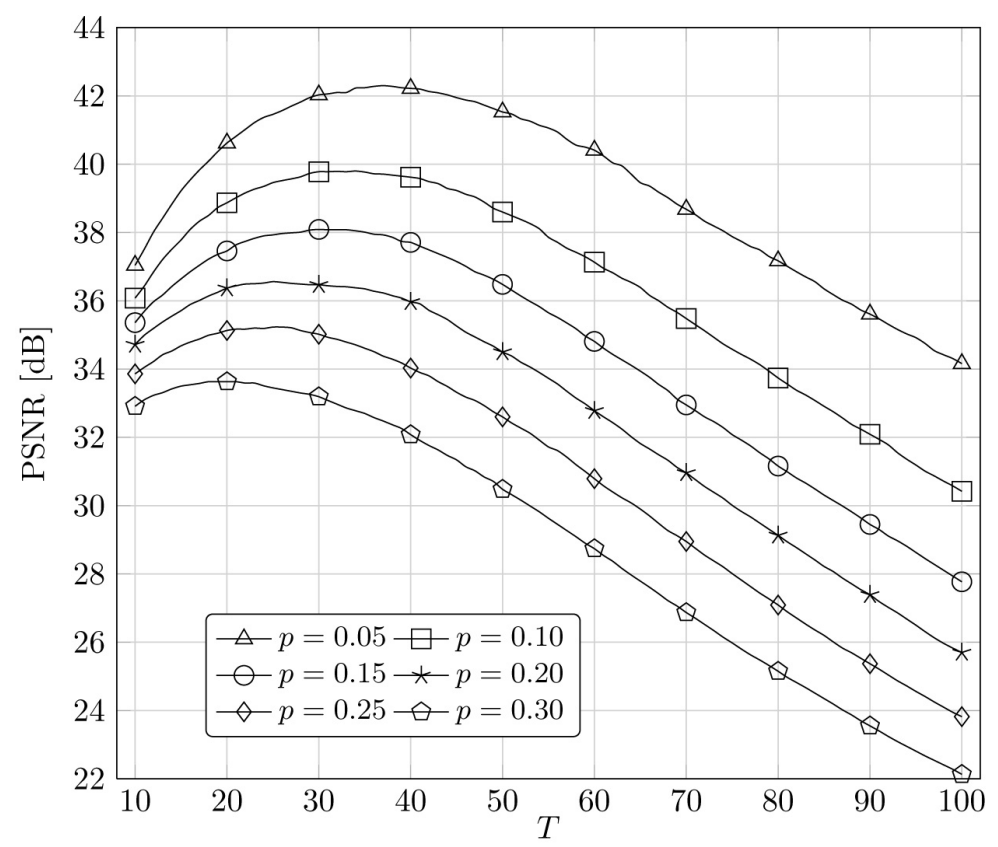

Fig. 4. Dependence of the PSNR on the threshold $T$ for $k=2$ obtained using the color test image LENA

In order to decide whether a pixel of a color image is corrupted by impulsive noise, the difference between the cumulated weighted distance $D_{1}$ assigned to the central pixel of the filtering window and the value of $D_{(1)}$ corresponding to the rank weighted vector median filter output can be used [29 31] and the strength of the impulsive contamination can be estimated as the difference between $D_{1}$ and $D_{(1)}$. In other words, the difference $\delta$ between the sum of the $k$ smallest distances to the neighbors of the central pixel of $W$ and the smallest sum of $k$ distances assigned to a pixel from $W$ will be treated as a measure of the pixel's impulsiveness 


$$
\delta=\sum_{r=1}^{k} \rho_{i(r)}-\sum_{r=1}^{k} \rho_{v(r)}=\sum_{r=1}^{k}\left(\rho_{i(r)}-\rho_{v(r)}\right),
$$

where $v$ is the position of the pixel in $W$ for which the sum of the $k$ smallest distances to the pixels of $W$ is minimized. Of course $\boldsymbol{x}_{v}$ is the output of the SVMF defined through (4) and (6).

Figure 1 depicts the noisy test image contaminated by impulsive noise (a), values of the sum of $k$ smallest distances $D_{1}$ assigned to the central pixel of the filtering window (b) and the smallest value $D_{(1)}(\mathrm{c})$. The differences $\delta=D_{1}-D_{(1)}$ depicted in (d) serve as measures of impulsive noise contamination. It can be observed that the map of the detected impulses correlates well with the real contamination.

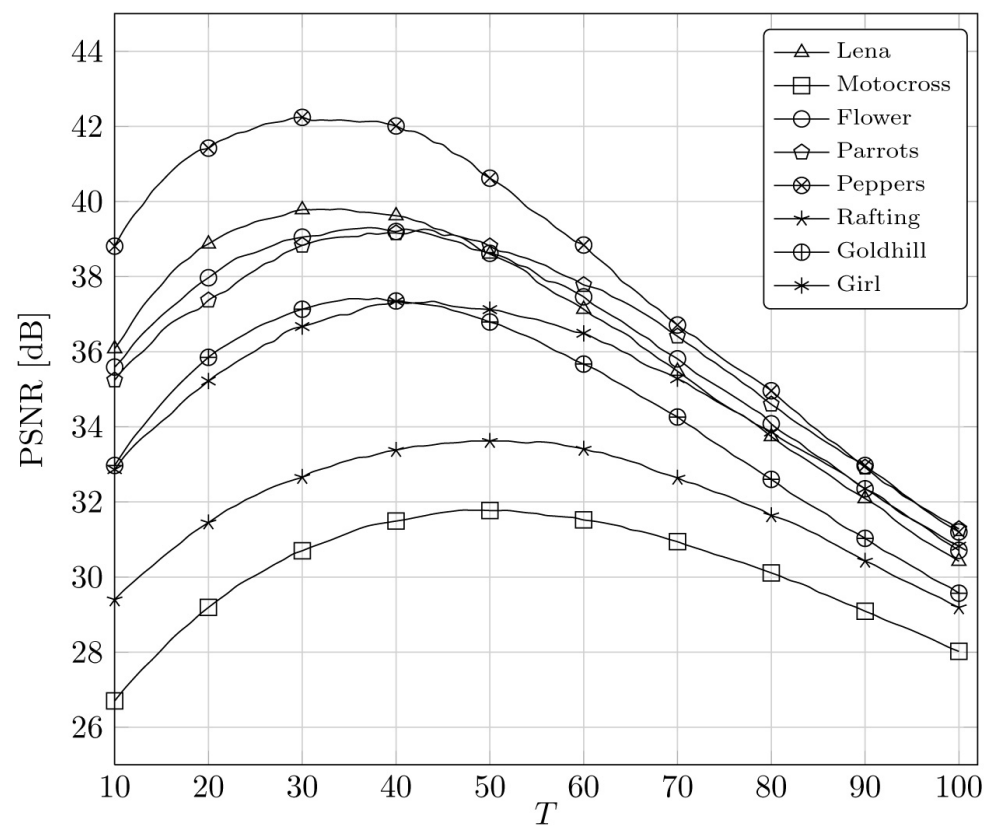

Fig. 5. Dependence of the PSNR on the thresholding parameter $T$ for various images depicted in Fig. 2 contaminated by impulsive noise of intensity $p=0.1$

The structure of the proposed switching filter is quite simple. If the difference $\delta=D_{1}-D_{(1)}$ exceeds a threshold value $T$, then a pixel is declared as corrupted by a noise process, otherwise it is treated as not disturbed.

$$
\boldsymbol{y}_{1}= \begin{cases}\hat{\boldsymbol{x}}, & \text { if } \delta>T \\ \boldsymbol{x}_{1}, & \text { otherwise }\end{cases}
$$


where $\boldsymbol{y}_{1}$ is the switching filter output, $\boldsymbol{x}_{1}$ is the central pixel of the filtering window and $\hat{\boldsymbol{x}}$ is the mean of the the pixels declared by the detector as not corrupted by the noise process. If all neighbors of the central pixel $\boldsymbol{x}_{1}$ of the filtering window are found to be corrupted, then the SVMF applied to all pixels from $W$ is taken as the filter output.

\section{$3 \quad$ Filter Parameters}

For the evaluation of the optimal parameters $k$ and $T$ of the proposed filtering technique, color test images depicted in Fig. 2 were corrupted by a noised modelled as

$$
\boldsymbol{x}_{i}= \begin{cases}\boldsymbol{v}_{i} & \text { with probability } p, \\ \boldsymbol{o}_{i} & \text { with probability } 1-p,\end{cases}
$$

where $\boldsymbol{x}_{i}$ is the pixel of the polluted image, $\boldsymbol{o}_{i}$ is the original sample, $\boldsymbol{v}_{i}$ is the corrupted pixel, whose components $\left\{v_{i 1}, v_{i 2}, v_{i 3}\right\}$ take on independent random values from the interval $[0,255]$, following the uniform distribution.

For the measurement of the restoration quality, the commonly used Root Mean Squared Error (RMSE) expressed through the Peak Signal to Noise Ratio (PSNR) was used, as the RMSE is a satisfactory measure of the efficiency of impulsive noise suppression and correlates well with other commonly used restoration quality measures [1].

The first parameter of the filter which needs to be determined is the number $k$ of the pixels whose distance is taken to build the cumulated sum of distances. Figure 3 depicts the dependence of the PSNR restoration quality measure on the number of close pixels $k$, when adjusting the thresholding parameter $T$, so that the best possible result is obtained. As can be seen, the setting $k=2$ is optimal in terms of the PSNR value. The low value of $k$ is very beneficial, as only two smallest distances are needed for the estimation of the degree of pixel distortion.

Of course, the efficiency of the switching scheme depends also on the value of the thresholding parameter. If the threshold $T$ is too low, the filter will be replacing undistorted pixels. On the other hand, if it is too high, many corrupted pixels will pass the filter without being replaced by the mean of the uncorrupted pixels.

Figure 4 shows the dependence of the PSNR on the threshold parameter $T$ using the LENA test image contaminated by impulsive noise with intensity ranging from 0.05 to 0.3 . As can be observed the optimal value of $T$ does not depend significantly on the contamination intensity. This behavior was also observed for other test image depicted in Fig. 2. For $k=2$ satisfactory results are obtained for $T=40$ and this setting was used as a default value used for comparisons with other filtering designs.

As can be observed in Fig. 5 the default value of the threshold is not significantly dependent on the image structures, which guarantees satisfactory filter behavior for natural images. 


\section{Comparison with Existing Techniques}

The proposed technique was compared with a various filters intended for the suppression of impulsive noise in color images. Their parameters were set according to the recommendations provided in the appropriate references [32, 33].

The following filters were chosen for the comparison:

- Peer Group Filter (PGF) 22],

- Adaptive Center-Weighted VMF (ACWVMF) 34],

- Adaptive Center-Weighted Directional-Distance Filter (ACWDDF) [35],

- Rank-Ordered VMF (ROVMF) [16],

- Sigma Directional Distance Filter (SDDF) 20, 19],

- Adaptive Vector Median Filter (AVMF) 7],

- Adaptive Basic Vector Directional Filter (ABVDF) [18],

- Sigma Vector Median Filter (SVMF) [19, 20] and

- Fast Modified Vector Median Filter (FMVMF) [8, 9].

Table 1. Comparison of the PSNR values obtained when restoring the color test images contaminated with impulsive noise using the proposed technique and methods known from the literature

\begin{tabular}{|c|c|c|c|c|c|c|c|c|c|c|c|}
\hline 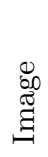 & $p$ & 空 & 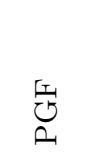 & 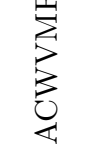 & 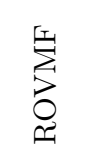 & 帘 & 鸽 & $\sum_{\text {䒚 }}$ & $\stackrel{\frac{1}{3}}{\stackrel{9}{4}}$ & 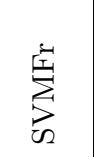 & $\sum_{\sum_{I}}^{\sum_{1}^{1}}$ \\
\hline \multirow{3}{*}{ 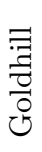 } & 0.1 & 37.13 & 36.19 & 35.47 & 35.54 & 33.94 & 34.94 & 34.74 & 28.39 & 33.87 & 32.39 \\
\hline & 0.2 & 34.17 & 32.66 & 31.07 & 32.26 & 30.53 & 29.57 & 31.86 & 25.23 & 29.37 & 30.42 \\
\hline & 0.3 & 31.38 & 29.14 & 26.29 & 28.52 & 26.84 & 24.13 & 28.39 & 22.63 & 24.24 & 27.67 \\
\hline \multirow{3}{*}{ 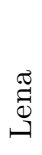 } & 0.1 & 39.78 & 38.56 & 37.87 & 38.12 & 36.34 & 36.07 & 37.28 & 33.17 & 36.18 & 35.50 \\
\hline & 0.2 & 36.47 & 34.54 & 31.93 & 33.98 & 31.69 & 29.46 & 33.46 & 29.01 & 29.85 & 32.66 \\
\hline & 0.3 & 33.19 & 30.29 & 26.62 & 29.65 & 27.03 & 23.75 & 29.50 & 25.11 & 24.18 & 29.10 \\
\hline \multirow{3}{*}{ 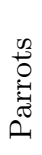 } & 0.1 & 38.83 & 37.32 & 36.73 & 36.29 & 36.78 & 36.70 & 35.79 & 29.11 & 35.73 & 34.82 \\
\hline & 0.2 & 36.00 & 33.98 & 31.37 & 33.09 & 31.83 & 29.82 & 32.66 & 25.76 & 29.39 & 32.27 \\
\hline & 0.3 & 32.73 & 29.43 & 26.03 & 28.59 & 26.73 & 23.81 & 28.39 & 22.84 & 23.77 & 28.25 \\
\hline \multirow{3}{*}{ 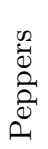 } & 0.1 & 42.24 & 37.56 & 36.71 & 37.07 & 35.09 & 34.36 & 36.75 & 32.54 & 34.75 & 34.71 \\
\hline & 0.2 & 38.50 & 33.42 & 30.79 & 32.75 & 30.27 & 27.59 & 32.53 & 27.72 & 28.58 & 31.54 \\
\hline & 0.3 & 34.03 & 28.58 & 25.10 & 27.80 & 24.89 & 21.98 & 27.82 & 22.82 & 22.79 & 27.30 \\
\hline
\end{tabular}



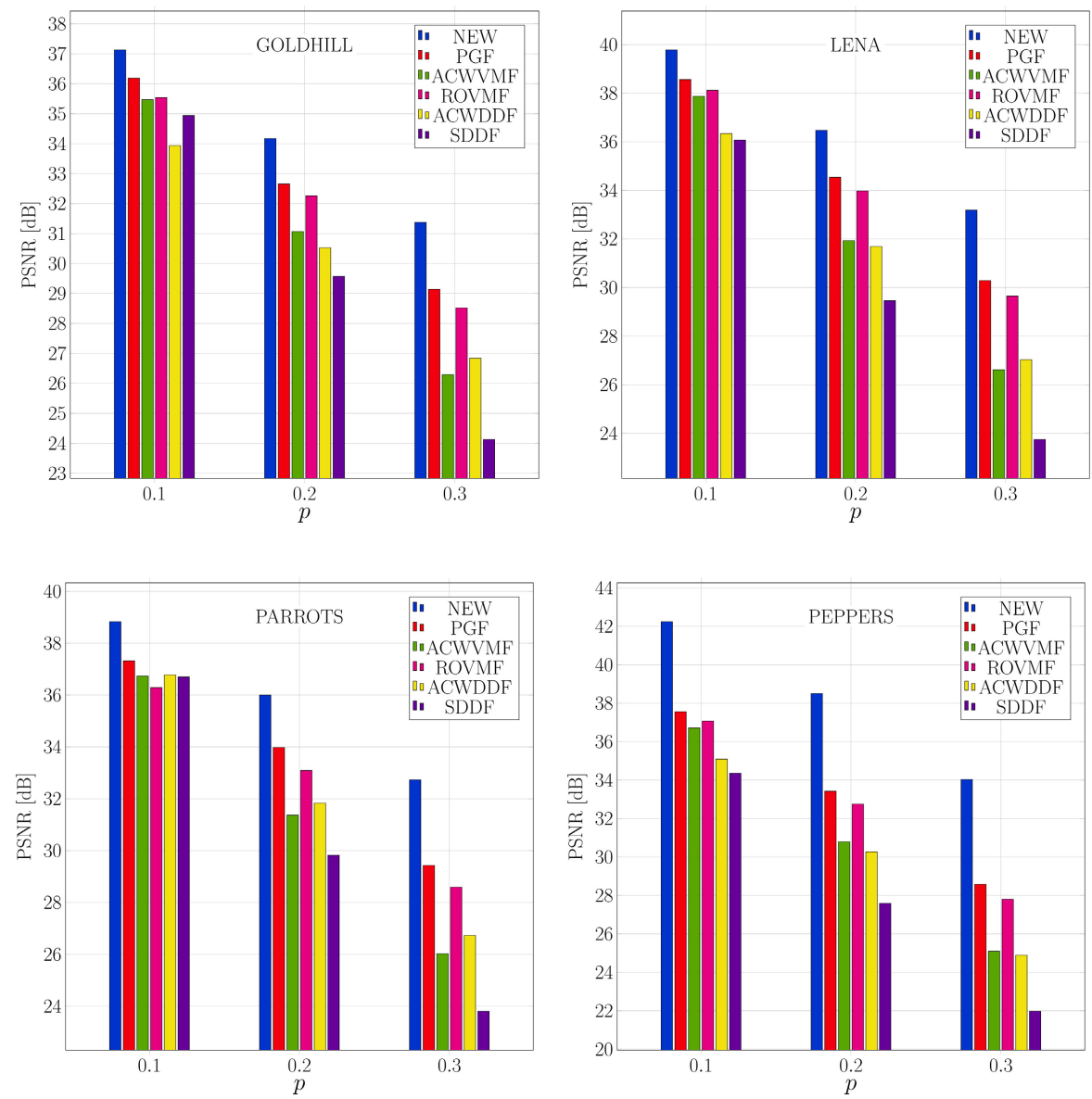

Fig. 6. Visualization of the results shown in Tab. 1 using the most efficient competitive filtering methods

The four images GOLDHILL, LENA, PARROTS and PEPPERS depicted in Fig. 2 were contaminated by the impulsive noise with intensities ranging from 0.05 to 0.3 . The obtained results are summarized in Tab. 1, Due to the space limitations only the PSNR measures are reported, however the results lead to the same conclusions when using other quality restoration measure like Mean Absolute Error (MAE) or Normalized Color Distance (NCD) in Lab color space [1]. 
As can be observed the proposed filtering method yields results significantly superior to those obtained using the state-of-the-art denoising methods. The results summarized in Tab. 1 and shown in a different form in Fig. 6] in which the best filters were chosen for comparisons, are confirmed by the visual comparison of the results depicted in Fig. 7 and Fig. 8, which show the restoration quality achieved using the proposed filter.

A beneficial feature if the new algorithm of impulsive noise suppression is its low computational complexity. For each pixel in the filtering, only the sum of distances to 2 nearest neighbors has to be calculated, which are then used to determine the measure of impulsiveness. Thus, the algorithm is comparable in terms of computational speed to the well known vector median filter, which needs the calculation of the sum of 8 distances, to all other pixels in $W$.

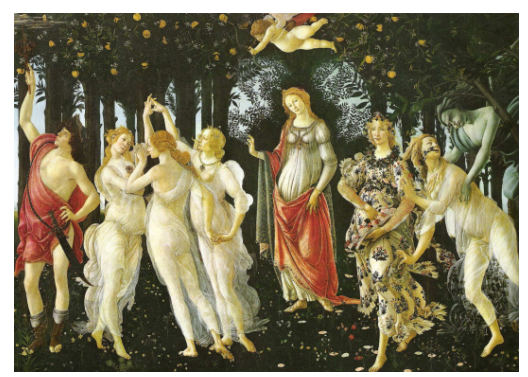

(a) La Primavera

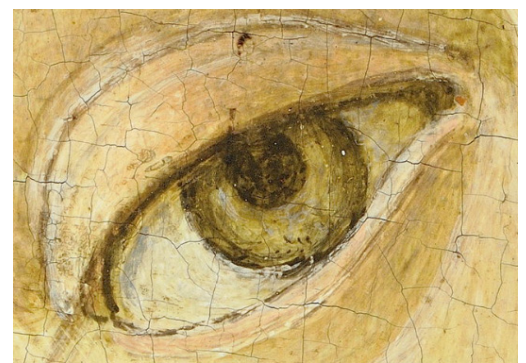

(c) original right eye

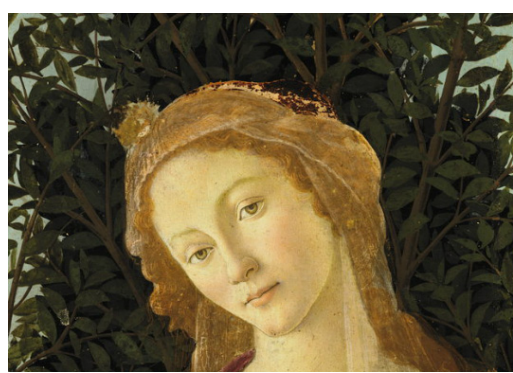

(b) face of the Venus

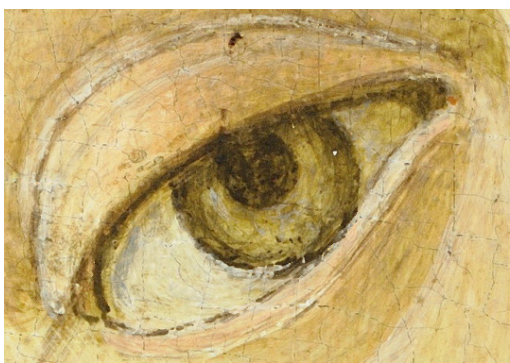

(d) restoration result

Fig. 7. Illustration of the effectiveness of the proposed restoration technique using the painting La Primavera by Sandro Botticelli 


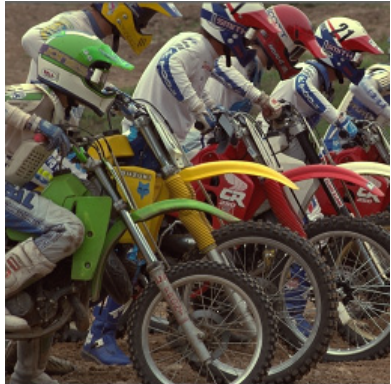

(a) original

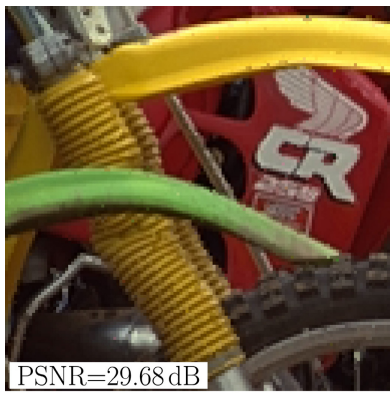

(d) PGF

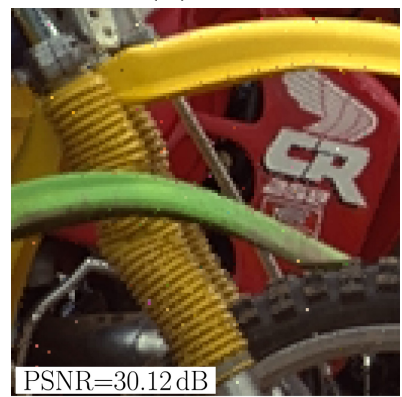

(g) ACWDDF

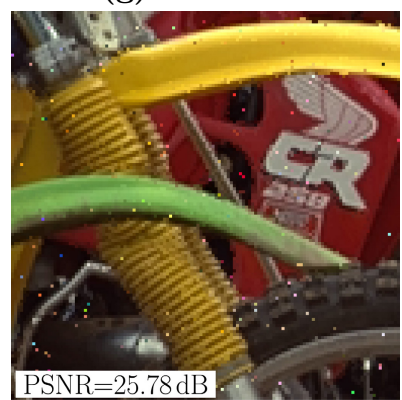

(j) ABVDF

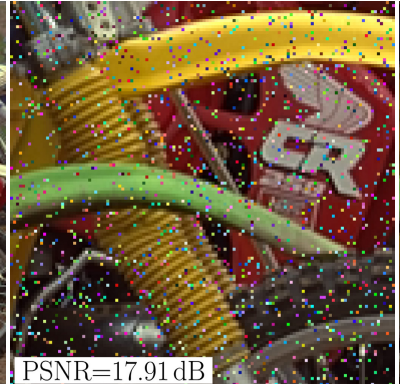

(b) noisy

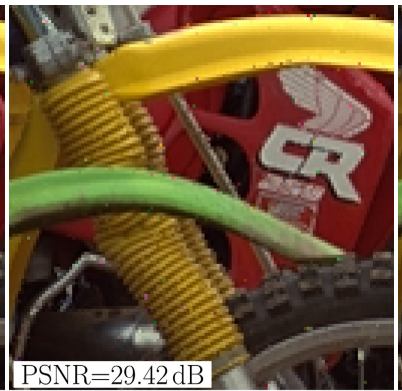

(e) ACWVMF

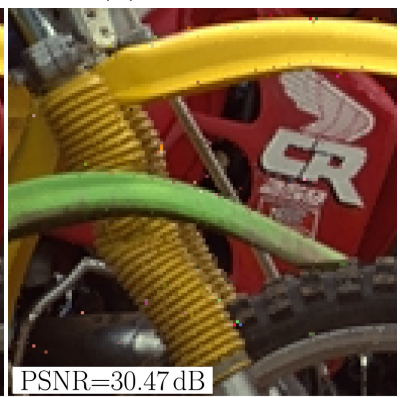

(h) SDDF

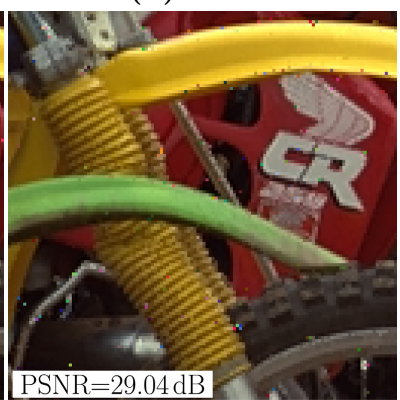

(k) SVMFr

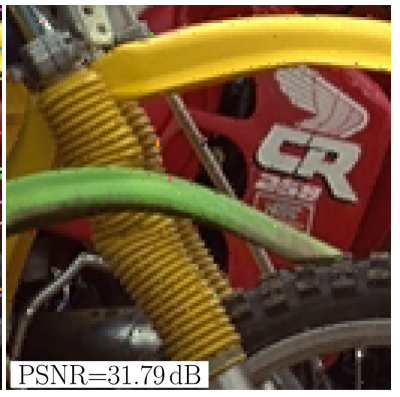

(c) PROPOSED

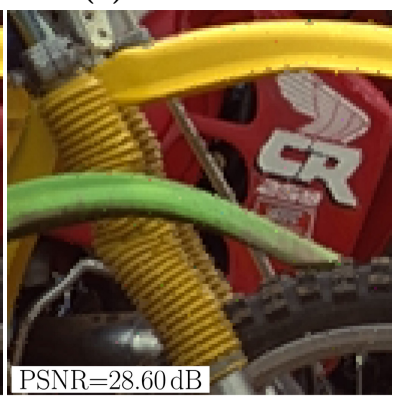

(f) ROVMF

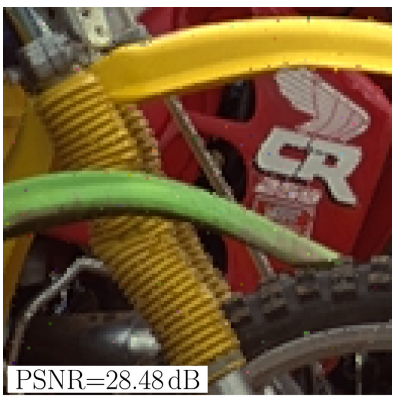

(i) AVMF

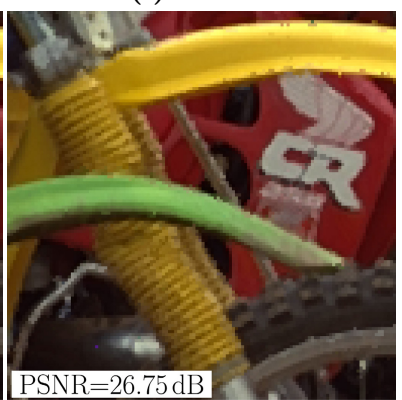

(1) FMVMF

Fig. 8. Comparison of the efficiency of the proposed switching filter as compared with the standard methods, using the color MOTORCROSS image corrupted by impulsive noise with intensity $p=0.1$ 


\section{Conclusions}

In the paper a novel switching filtering design intended for the removal of impulsive noise in color images has been proposed. The described filter is based on the trimmed cumulative distances between pixels, which are used for the detection of samples corrupted by impulses.

The experiments performed on natural, color test images confirmed the high efficiency of the proposed filtering framework. Comparison with existing techniques revealed that the new filter offers excellent efficiency of the impulsive noise suppression while better preserving image details. Moreover, the novel filter has a low computational complexity and simple structure, which makes it attractive for real-time applications. Further research will be focused on the construction of an adaptive design, capable of tuning the filter's parameter to the intensity of the noise corruption.

Acknowledgement. This work has been supported by the Polish Ministry of Science and Higher Education development grant: Nonlinear methods of noise reduction in color images and video sequences, (registration number 2012/05/B/ ST6/03428).

\section{References}

1. Plataniotis, K., Venetsanopoulos, A.: Color Image Processing and Applications. Springer (2000)

2. Lukac, R., Smolka, B., Martin, K., Plataniotis, K., Venetsanopoulos, A.: Vector filtering for color imaging. IEEE Signal Processing Magazine 22(1), 74-86 (2005)

3. Boncelet, C.G.: Image noise models. In: Bovik, A. (ed.) Handbook of Image and Video Processing, pp. 325-335. Academic Press (2000)

4. Astola, J., Haavisto, P., Neuvo, Y.: Vector median filters. Proceedings of the IEEE 78(4), 678-689 (1990)

5. Smolka, B., Plataniotis, K., Venetsanopoulos, A.: Nonlinear techniques for color image processing. In: Nonlinear Signal and Image Processing: Theory, Methods, and Applications, pp. 445-505. CRC Press (2004)

6. Smolka, B., Venetsanopoulos, A.: Noise reduction and edge detection in color images. In: Color Image Processing: Methods and Applications, pp. 75-100. CRC Press (2007)

7. Lukac, R.: Adaptive vector median filtering. Pattern Recognition Letters 24(12), 1889-1899 (2003)

8. Smolka, B., Chydzinski, A., Wojciechowski, K.W., Plataniotis, K.N., Venetsanopoulos, A.N.: On the reduction of impulsive noise in multichannel image processing. Optical Engineering 40(6), 902-908 (2001)

9. Smolka, B., Plataniotis, K.N., Chydzinski, A., Szczepanski, M., Venetsanopoulos, A.N., Wojciechowski, K.: Self-adaptive algorithm of impulsive noise reduction in color images. Pattern Recognition 35(8), 1771-1784 (2002)

10. Smolka, B., Chydzinski, A.: Fast detection and impulsive noise removal in color images. Real-Time Imaging 11(5-6), 389-402 (2005); Special Issue on MultiDimensional Image Processing 
11. Smolka, B.: Peer group switching filter for impulse noise reduction in color images. Pattern Recognition Letters 31(6) (2010)

12. Ma, Z., Feng, D., Wu, H.: A neighborhood evaluated adaptive vector filter for suppression of impulse noise in color images. Real-Time Imaging 11(5-6), 403-416 (2005)

13. Morillas, S., Gregori, V., Peris-Fajarneés, G., Latorre, P.: A fast impulsive noise colour image filter using fuzzy metrics. Real-Time Imaging 11(5) (2005)

14. Morillas, S., Gregori, V., Peris-Fajarnés, G., Sapena, A.: Local self-adaptive fuzzy filter for impulsive noise removal in color images. Signal Processing 88(2), 390-398 (2008)

15. Celebi, M., Kingravi, H., Uddin, B., Asl, Y.: A fast switching filter for impulsive noise removal from color images. Journal of Imaging Science and Technology (2007)

16. Peris-Fajarnés, G., Roig, B., Vidal, A.: Rank-Ordered Differences Statistic Based Switching Vector Filter. In: Campilho, A., Kamel, M.S. (eds.) ICIAR 2006. LNCS, vol. 4141, pp. 74-81. Springer, Heidelberg (2006)

17. Garnett, R., Huegerich, T., Chui, C., Wenjie, H.: A universal noise removal algorithm with an impulse detector. IEEE Transactions on Image Processing 14(11), 1747-1754 (2005)

18. Lukac, R.: Color image filtering by vector directional order-statistics. Pattern Recognition and Image Analysis 12, 279-285 (2002)

19. Lukac, R., Smolka, B., Plataniotis, K., Venetsanopoulos, A.: Vector sigma filters for noise detection and removal in color images. Journal of Visual Communication and Image Representation 17(1), 1-26 (2006)

20. Lukac, R., Plataniotis, K.N., Venetsanopoulos, A.N., Smolka, B.: A statisticallyswitched adaptive vector median filter. Journal of Intelligent and Robotic Systems 42(4), 361-391 (2005)

21. Deng, Y., Kenney, C., Moore, M.S., Manjunath, B.S.: Peer group filtering and perceptual color image quantization. In: Proceedings of IEEE International Symposium on Circuits and Systems, vol. 4, pp. 21-24. Springer (1999)

22. Kenney, C., Deng, Y., Manjunath, B.S., Hewer, G.: Peer group image enhancement. IEEE Trans. on Image Processing 10(2), 326-334 (2001)

23. Morillas, S., Gregori, V., Hervas, A.: Fuzzy peer groups for reducing mixed gaussian-impulse noise from color images. IEEE Transactions on Image Processing 18(7), 1452-1466 (2009)

24. Ho, J.: Peer region determination based impulsive noise detection. In: Proceedings of ICASP, vol. 3, pp. 713-716 (2003)

25. Ma, Z., Wu, H.R., Qiu, B.: A window adaptive hybrid vector filter for color image restoration. In: Proceedings of ICASSP, vol. 3, pp. 205-208 (2004)

26. Morillas, S., Gregori, V., Peris-Fajarnés, G.: Isolating impulsive noise pixels in color images by peer group techniques. Computer Vision and Image Understanding 110(1), 102-116 (2008)

27. Camarena, J., Gregori, V., Morillas, S., Sapena, A.: Fast detection and removal of impulsive noise using peer groups and fuzzy metrics. Journal of Visual Communication and Image Representation 19(1), 20-29 (2008)

28. Lukac, R., Smolka, B., Sharpening, K.P.: vector median filters. Signal Processing 87, 2085-2099 (2007)

29. Smolka, B.: Adaptive Edge Enhancing Technique of Impulsive Noise Removal in Color Digital Images. In: Schettini, R., Tominaga, S., Trémeau, A. (eds.) CCIW 2011. LNCS, vol. 6626, pp. 60-74. Springer, Heidelberg (2011) 
30. Smolka, B.: Adaptive rank based impulsive noise reduction in color images. In: IEEE International Conference on Communications and Electronics (ICCE 2012), Hue, Vietnam, pp. 355-359 (2012)

31. Smolka, B.: Adaptive truncated vector median filter. In: IEEE International Conference on Computer Science and Automation Engineering (CSAE 2012), Shanghai, China, pp. 261-266 (2011)

32. Lukac, R., Plataniotis, K.: A taxonomy of color image filtering and enhancement solutions. Advances in Imaging and Electron Physics, vol. 140, pp. 187-264. Elsevier (2006)

33. Celebi, M., Kingravi, H., Aslandogan, Y.: Nonlinear vector filtering for impulsive noise removal from color images. J. Electron. Imaging 16(3), 033008 (2007)

34. Lukac, R.: Adaptive color image filtering based on center-weighted vector directional filters. Multidimensional Systems and Signal Processing 15(2), 169-196 (2004)

35. Lukac, R., Smolka, B., Plataniotis, K.N., Venetsanopulos, A.N.: Selection weighted vector directional filters. Computer Vision and Image Understanding 94(1-3), 140-167 (2004) 